\title{
Two-dimensional Chebyshev Polynomials for Image Fusion
}

\author{
Zaid Omar \\ Faculty of Electrical Engineering, \\ Universiti Teknologi Malaysia, \\ Johor Bahru, \\ Malaysia \\ zaid@fke.utm.my
}

\author{
Nikolaos Mitianoudis, Tania Stathaki \\ Communications and Signal Processing Group, \\ Imperial College London, \\ South Kensington, \\ United Kingdom \\ n.mitianoudis, t.stathaki@imperial.ac.uk
}

\begin{abstract}
This paper presents a novel method for fusing images in a domain concerning multiple sensors and modalities. Using Chebyshev polynomials as basis functions, the image is decomposed to perform fusion at feature level. Results show favourable performance compared to previous efforts on image fusion using ICA. The work presented here aims at providing a novel framework for future studies in image analysis and may introduce innovations in the fields of surveillance, medical imaging and remote sensing.
\end{abstract}

Keywords: Image and data fusion, Chebyshev polynomials, orthogonal moments.

\section{Introduction}

Images may be acquired from various types of sources. These may consist of different sensor modalities or just particular aspects of the image scene. Several real-time computer vision tasks, such as person tracking and weapon detection, can not rely on single-modality sensors in order to extract the required information within a scene[1]. Therefore, we have witnessed the emergence of multimodal image acquisition platforms to be used in computer vision tasks. Image data from two or more sources can be mathematically merged to generate a superior composite output, so as to reduce errors and improve the accuracy and performance of these systems. Image Fusion is a general framework that is employed to perform the above task.

Fusion is usually performed at feature level in a transform domain. Popular transformations for image fusion are the Wavelet transform and the Laplacian pyramid decomposition [1]. Independent Component Analysis (ICA) [2] trains gabor-likely statistically independent basis vectors, offering custom transformations for different image groups that can improve fusion performance compared to wavelet approaches [3]. In real-life surveillance conditions, the acquired images are often contaminated by noise or other disturbances. Wavelet-based or ICA-based sparse code shrinkage has been efficiently used to tackle the denoising problem $[2,1]$. However, the level (variance) of noise needs to be evaluated before performing denoising using sparse code shrinkage. This gives rise to a novel approach of using twodimensional Chebyshev polynomials for image fusion with inherent noise reduction features[4], due to their smooth feature extraction property. Due to the fast convergence of Chebyshev series, the method is expected to achieve faster performance compared to ICA-based methods.

The first section introduces the application and rationale of Image Fusion and the motivation behind using Chebyshev polynomials for fusion. Section 2 elaborates the state of the art regarding image and data fusion technology. The third section revises the basics of Chebyshev polynomial theory and its use in signal approximation. Section 4 describes the fusion method and rules that will be applied and section 5 evaluates the performance against incumbent methods in image fusion.

\section{Theory of data fusion}

There is currently a wide range of techniques for capturing image and scenery,including infra-red, laser and camera, ladar, magnetic resonance (MRI), optical and particle image velocimetry (PIV) [11]. The technologies differ in terms of instrument modalities, acquisition techniques, information characteristics (thermal, visual) and errors (imprecision, ambiguity, incompleteness); therefore the extracted information is often different and complementary in nature. The images may also differ in terms of object focus, colour, texture and spectral representation. The fusion process aims to combine the best aspects of each modality in order to either produce a better looking image for subjective validation and further computer analysis or make decisions based on the fused dataset.

In critical tracking applications, decisions pertaining the system conditions are very rarely made upon the measurements of a single parameter. Often these decisions are based on the inputs of multiple parameters from different sensors, so as to reduce the risk of error. The advantages of multiple-sensor over single-sensor systems are numerous, such as higher SNR, increased robustness and reliability, improved resolution and reduction in measurement time and costs [11]. 
In this paper, we will be following the Waterfall model [11]. The system operates on three different levels of abstraction - raw data, features and decision making. As it can be seen from Figure 1, a continuous feedback loop exists from the decision output to the sensors that enables reconfiguration and re-alignment of the data acquisition process.

Figure 1: Waterfall model [11]

The low level representation stage consists of a sensor system that is gathering and pre-processing real-world data. Data is further analysed (transformation, feature extraction) in order to enhance the information content and omit redundancies. Data are fused in the middle level. The fused output is then forwarded to the decision making stage, where possible action routes are considered according to the available data, e.g. system information and user interactions.

Diligent efforts have been made by the data fusion community to address the confusion regarding terminologies in the area of fusion. In [15], data fusion is generally categorised into low- (LLF), intermediate- (ILF) and high-level fusion (HLF). LLF fuses raw data directly from sources, while ILF combines in a sophisticated manner features that have been extracted from data. ILF is also known as features fusion. HLF tackles fusion in the decision making level, where various confidence measures are taken into account in making one penultimate decision.

\section{Chebyshev polynomials}

\subsection{One-dimensional Chebyshev polynomials}

Tchebichef or Chebyshev polynomials (CP) of the first kind, are defined as the mathematical solution to the Chebyshev differential equation

$$
\left(1-x^{2}\right) y^{\prime \prime}-x y^{\prime}+n^{2} y=0
$$

where $n$ is a non-negative integer. The polynomials can be recursively generated i.e.

$$
T_{0}(x)=1, T_{1}(x)=x, T_{n+1}(x)=2 x T_{n}(x)-T_{n-1}(x) .
$$

The first ten CP's are given below,

$$
\begin{aligned}
& T_{0}(x)=1 \\
& T_{1}(x)=x \\
& T_{2}(x)=2 x^{2}-1 \\
& T_{3}(x)=4 x^{3}-3 x \\
& T_{4}(x)=8 x^{4}-8 x^{2}+1 \\
& T_{5}(x)=16 x^{5}-20 x^{3}+5 x \\
& T_{6}(x)=32 x^{6}-48 x^{4}+18 x^{2}-1 \\
& T_{7}(x)=64 x^{7}-112 x^{5}+56 x^{3}-7 x \\
& T_{8}(x)=128 x^{8}-256 x^{6}+160 x^{4}-32 x^{2}+1 \\
& T_{9}(x)=256 x^{9}-576 x^{7}+432 x^{5}-120 x^{3}+9 x
\end{aligned}
$$

Chebyshev polynomials are orthogonal with respect to the weight function $W(x)=\frac{1}{\sqrt{1-x^{2}}}$ and valid over the interval $[-1,1]$. Because CP is orthogonal, the solution of a mathematical equation can be reduced to a simple inner product, as explained in [14]. In all instances, the local extrema of CP tends to be either 1 or -1 , such as in Figure 2. The stable values as compared to other orthogonal functions are a mitigating factor of its application in signal approximations. Trigonometric identity functions may also be used to define CP [5].

Figure 2: Chebyshev polynomials, up to $N=5$ degrees (image taken from www.wikipedia.org) 


\subsection{Chebyshev polynomials for signal ap- proximation}

Approximating a function is theoretically done with another function that utilises the arithmetic operation of basis components, in our case comprising of mathematical polynomials. Approximation aims to recreate an estimate that is as close as possible to its original, which means finding the minimal mean square error (MSE) of the estimate. A small loss of data and the perceived quality of the result implies a good estimation.

Noise, whether Gaussian or speckled, are often viewed as abrupt and random interferences in a signal. Chebyshev Polynomial (CP) approximation is able to overcome this. The expansion of Chebyshev approximation $\tilde{f}(x)$ is considered important, as errors are spread smoothly across the interval $-1 \leq x \leq 1$. Due to this property, the approximation of Chebyshev polynomials will result in a smoother function that purges any abrupt abnormalities in the signal, thus effectively reducing noise.

CPs are generally compact and a small degree $n$ is often required to approximate a signal sufficiently. Furthermore, CPs are known a priori and can be applied regardless of the signal type. In contrast, ICA bases perform better with the specific type of images, they were trained for. Thus, for a specific type of image, one has to train a set of ICA bases in advance, so as to benefit from their performance. Compared to other orthogonal polynomials (Zernike, Legendre), Chebyshev converge faster and require no discrete transformation. Hence, they are not prone to the continuous-discrete error, compared to other polynomials series [10].

The expansion of the Chebyshev series [6] form a complete basis set in a Sobolev space and is used to model almost any function through the formula

$$
\tilde{f}(x)=\sum_{n=0}^{N} a_{n} T_{n}(x)
$$

where $a_{n}$ is a coefficient of $n$ and defined by

$$
a_{n}=\frac{2}{\pi} \sum_{x=-1}^{1}(1-x)^{-\frac{1}{2}} f(x) T_{n}(x)
$$

while $f(x)$ is a general function and $\tilde{f}(x)$ is the approximated function. A calculation of the MSE defines the CP approximation quality.

The approximation by Chebyshev polynomials is controlled primarily by two parameters - data dimension $L$ (length of data to be approximated) and also the limit of polynomial degree $n$. An optimal estimation requires a balanced choice for both variables. In general, the more CPs the better their approximation, but this would also increase the processing time. Very large-order CPs, for example above 100 , may cause instability and error and would then require an orthonormal version of CPs [12], increasing the complexity. Equally important is the choice of data size $L$. A small data size $L$ may not summarise the overall function
(a) $\mathrm{L}=12$
(b) $\mathrm{L}=35$
(c) $\mathrm{L}=100$

Figure 3: Chebyshev polynomial approximation in one variable $(f(x)$ in blue, $\tilde{f}(x)$ in green)

correctly, whilst a large $L$ may result into a non-stationary signal that is too complex to model.

Figure 3 demonstrates the Chebyshev polynomial approximation for a single variable, using a 10-th order CP ( $n=$ 10). As it can be seen in Figure 3(c), the small number $n$ of CPs compared to the large data size $L$ results in a rather primitive approximation of the signal. In Figure 3(a), a better signal approximation is obtained when the $\frac{n}{L}$ ratio is high and thus the signal is more accurately reconstructed.

\subsection{Chebyshev polynomial approximation with two variables}

Chebyshev polynomials can be extended to more than one variables [7]. This paper is concerned with the study of CPs for images, analysis and approximation, i.e. 2D functions.

\subsubsection{Two-dimensional Chebyshev polynomials as basis functions}

In 1974, a two-dimensional description of the classical Chebyshev polynomial of the second kind $P_{k, l}$ was first introduced by Koornwinder [8] and its properties were elaborated in [7]. This concept was pursued in [9], where the definitions of the 2D CP were introduced.

$$
\begin{gathered}
P_{-1, l}(z, \bar{z})=0, P_{k,-1}(z, \bar{z})=0 \\
P_{0,0}(z, \bar{z})=1 \\
P_{1,0}(z, \bar{z})=z, P_{0,1}(z, \bar{z})=\bar{z}
\end{gathered}
$$

A more general form is the following:

$z P_{k, l}(z, \bar{z})=P_{k+1, l}(z, \bar{z})+P_{k-1, l+1}(z, \bar{z})+P_{k, l-1}(z, \bar{z})$
$\bar{z} P_{k, l}(z, \bar{z})=P_{k, l+1}(z, \bar{z})+P_{k+1, l-1}(z, \bar{z})+P_{k-1, l}(z, \bar{z})$

where $z=x+j y$ and $\bar{z}=x-j y$ are complex correspondents of $x$ and $y$. The following definition establishes two-variable Chebyshev polynomials as basis functions for 2D signal approximation

$$
\tilde{f}(z, \bar{z})=\sum_{k=0}^{K} \sum_{l=0}^{L} a_{k, l} P_{k, l}(z, \bar{z}) .
$$


The 2D signal $\tilde{f}(z, \bar{z})$ is a linear combination of $2 \mathrm{D}$ Chebyshev Polynomials. The problem is to calculate the coefficients $a_{k, l}$, which are given by

$$
a_{k, l}=\sum_{z=-1}^{1} \sum_{\bar{z}=-1}^{1} \mu(z, \bar{z}) f(z, \bar{z}) P_{k, l}(z, \bar{z})
$$

The approximation forms an orthonormal system subject to the weight function:

$$
\mu(z, \bar{z})=\frac{1}{2 \pi^{2}} \sqrt{-z^{2} \bar{z}^{2}+4 z^{3}+4 \bar{z}^{3}-18 z \bar{z}+27} .
$$

However, this entails that a system must reside over the region $S$ inside Steiner's hypocycloid, $\partial S$.

$$
\partial S(\theta)=2 e^{j \theta}+e^{-2 j \theta}, 0 \leq \theta \leq 2 \pi .
$$

To solve this, we will have to integrate the system over $0 \leq \theta \leq 2 \pi$ in the region above. However, orthogonal polynomials do not correspond to the discrete image coordinate space. Thus, we will require a transformation [10] of the Cartesian coordinate i.e. from $(x, y)$ to $(\theta, r)$ all within the boundaries of a hypocycloid shape. The complexity in solving this problem has led us to contemplate alternative definitions for two-dimensional Chebyshev polynomials.

\subsubsection{Separable Chebyshev polynomials as basis func- tions}

Over the years, research has been focused on the implementation of orthogonal moments (including Chebyshev polynomials) in image analysis [10]. These studies offer a different, but nonetheless valid form of the $2 \mathrm{D} \mathrm{CP}$, as it shall be proven.

Approximation of two-dimensional signals is achieved with two separable 1D Chebyshev polynomials $T(x)$ and $T(y)$ that are discrete and orthogonal, similar to the Discrete Cosine Transform (DCT) and Haar transform. This massively reduces the complexity, as the same principles from one-dimensional CP can now be applied, i.e. intervals of $x$ and $y$ are still between $[-1,1]$. We employ the same approximation algorithm and weight factor as those described in Section 3.2. Thus, the following 2D CP approximation of a signal is introduced:

$$
\tilde{f}(x, y)=\sum_{m=0}^{M} \sum_{n=0}^{N} a_{m, n} T_{m}(x) T_{n}(y),
$$

We are concerned with finding $a_{m, n}$. So if the left hand side is multiplied as below, using the orthogonality relation,

$$
\begin{array}{r}
A=\sum_{x=-1}^{1} \mu(x) f(x, y) T_{k}(x)= \\
\sum_{x=-1}^{1} \mu(x) \sum_{m=0}^{M} \sum_{n=0}^{N} a_{m, n} T_{m}(x) T_{n}(y) T_{k}(x)=
\end{array}
$$

$$
\sum_{m=0}^{M} \sum_{n=0}^{N} a_{m, n} T_{n}(y) \sum_{x=-1}^{1} \mu(x) T_{m}(x) T_{k}(x)
$$

We know that

$$
\sum_{x=-1}^{1} \mu(x) T_{m}(x) T_{k}(x)=\frac{\pi}{2} \delta(m-k)
$$

Given that $\delta(m-k)=0$ if $m \neq k$ and $\delta(m-k)=1$ if $m=k$, we now have

$$
A=\frac{\pi}{2} \sum_{n=0}^{N} a_{k, n} T_{n}(y)
$$

We now apply the transformation along $y$, i.e.

$$
\begin{aligned}
& \sum_{y=-1}^{1} \mu(y) \sum_{x=-1}^{1} \mu(x) f(x, y) T_{k}(x) T_{l}(y)= \\
& \frac{\pi}{2} \sum_{n=0}^{N} a_{k, n} \sum_{y=-1}^{1} \mu(y) T_{n}(y) T_{l}(y)=\frac{\pi^{2}}{4} a_{k, l}
\end{aligned}
$$

so finally we have

$a_{m, n}=\frac{4}{\pi^{2}} \sum_{x=-1}^{1} \sum_{y=-1}^{1} \mu(x) \mu(y) f(x, y) T_{m}(x) T_{n}(y)$.

Chebyshev approximation (sometimes referred to as Chebyshev moments) have employed for image analysis in the past, mainly for pattern recognition and image segmentation [13], image reconstruction and rendering [10]. This paper aims to apply the use Chebyshev polynomial approximation on image fusion.

\section{Image fusion with Chebyshev poly- nomials}

$\mathrm{CP}$ analysis is a form of transformation, where the necessary features are extracted to preserve the second-order image statistics. A finite $\mathrm{CP}$ expansion, (finite order $n$ ), ensures that only the prominent information are retained, while any redundant statistics are discarded [13]. Conversely, a higher order approximation is more precise, though several studies have noted that very high orders of CPs tend to cause numerical instabilities, which result in severe approximation errors. To overcome this, orthonormal Chebyshev moments have been proposed [12].

An example of image fusion using Chebyshev polynomials is described below. In this case, fusion is performed by comparing the coefficients of the two or more input images. As CPs are derived from a formula, they can be precomputed and stored in order to reduce processing complexity.

Let there be two image approximations,

$$
f_{1}(x, y)=\sum_{m=0}^{M} \sum_{n=0}^{N} \alpha_{m, n} T_{m}(x) T_{n}(y)
$$




$$
\begin{gathered}
f_{2}(x, y)=\sum_{m=0}^{M} \sum_{n=0}^{N} \beta_{m, n} T_{m}(x) T_{n}(y) \\
f_{\text {fused }}(x, y)=\sum_{m=0}^{M} \sum_{n=0}^{N} \max \left\{\left|\alpha_{m, n}\right|,\left|\beta_{m, n}\right|\right\} T_{m}(x) T_{n}(y)
\end{gathered}
$$

The fused image approximation $f_{\text {fused }}(x, y)$ is formed by fusing the two coefficients, via the $\max$-abs fusion rule, i.e. choose the coefficient with the higher absolute value. As each coefficient corresponds to the global image and information strength, performing max-abs over the two images will retain and enhance the strong pixels while suppressing the weak ones. This is essentially image fusion, resulting to an improved quality image. Several other fusion rules can also be considered, but for simplicity we will be using max$a b s$.

\section{Performance Evaluation}

Image fusion performance using Chebyshev polynomials is evaluated using a number of grayscale image inputs. For benchmarking purposes, the ICA-based fusion method using the "max-abs" rule [3] is also used. The methods are tested under several conditions, including images contaminated with noise and multi-modal examples. A $3 \times 3$ frame was used for the ICA bases, whilst $m=11$ and $n=11$ were used for the Chebyshev polynomials and the input image was divided into $3 \times 3$ overlapping patches (so as to minimise complexity). Larger data patches usually carry too much detail and will thus require a high degree of $\mathrm{CP}$ for more accurate approximation, which may lead to unstable results. The window size we used for both ICA and CP are deemed optimal for this experiment. There is also no need for image registration in these examples.

Figure 4 illustrates our results. Four different experiments in total were conducted. The resulting Figure 4(d) for CP compares favourably with 4(c) for ICA. Subsequently, one of the inputs is corrupted by Gaussian noise with mean $=0$ and variance $=0.001$, and the result appears significantly better for CP 4(h) than ICA 4(g). As stated previously, CP's smooth approximation is able to filter the disturbance caused by noise.

In the next example, multimodal images are tested. The CP result for the UN camp image in Figure 4(1) is slightly clearer than ICA in $4(\mathrm{k})$. Similarly, for medical images in 4(p), CP tends to have much stronger features than ICA in 4(o).

The results are also put under objective evaluation using the Petrovic and Piella fusion metrics respectively[1]. Table 1 validates our subjective assessment. CP-based fusion exhibits a slightly lower score $(-7 \%$ decrease in Petrovic, $-4 \%$ in Piella) for multifocus images in Figure 4(a) and 4(b), but performs much better against ICA in the test involving corrupted signals $(+21 \%$ Petrovic, $+28 \%$ Piella) in Figure 4(e). This is due to the automated denoising performed by CP approximation. Instead, ICA could perform denoising using sparse code shrinkage, but the vari- (a) Input 1 - back-(b) Input 2 - foreground focused ground focused

(c) ICA

(d) $\mathrm{CP}$

(e) Input 1 - noise corrupted

(f) Input 2

(g) ICA

(h) $\mathrm{CP}$

(i) Input 1 - visual(j) Input 2 - in-

(k) ICA

(1) $\mathrm{CP}$

camera frared camera

(m) Input 1 - MRI(n) Input 2 - CT scan

(o) ICA

(p) $\mathrm{CP}$

Figure 4: Fusion results - ICA versus CP

ance of noise needs to be defined before denoising can be performed. In the multimodal UN camp images, in Figures 4(i) and 4(j) images, CP scores lower ( $-6 \%$ Petrovic, $-15 \%$ Piella), though conversely in medical images in Figures $4(\mathrm{~m})$ and $4(\mathrm{n})$ its score is significantly better $(+13 \%$ Petrovic, $+12 \%$ Piella). Overall, for the categories in which ICA outperforms CP, the results as can be seen are close. However, those in which $\mathrm{CP}$ is better, the difference in scores is quite clear.

\begin{tabular}{|l|c|c|c|}
\hline Input & Method & Petrovic & Piella \\
\hline \multirow{2}{*}{ Multifocus clock } & ICA & $\mathbf{0 . 6 4 5 0}$ & $\mathbf{0 . 9 1 9 4}$ \\
& CP & 0.5980 & 0.8826 \\
\hline \multirow{2}{*}{ Noise corrupted clock } & ICA & 0.3717 & 0.5821 \\
& CP & $\mathbf{0 . 4 6 9 1}$ & $\mathbf{0 . 8 0 5 2}$ \\
\hline \multirow{2}{*}{ Multimodal UN camp } & ICA & $\mathbf{0 . 4 6 5 2}$ & $\mathbf{0 . 6 7 4 2}$ \\
& CP & 0.4349 & 0.5714 \\
\hline \multirow{2}{*}{ Multimodal medical image } & ICA & 0.4680 & 0.6698 \\
& CP & $\mathbf{0 . 5 3 7 1}$ & $\mathbf{0 . 7 5 8 1}$ \\
\hline
\end{tabular}

Table 1: Fusion metric evaluation - ICA versus CP 
We also compared the processing speed between the two algorithms. ICA requires pre-processing and training of basis components which take up time, and was slower compared to $\mathrm{CP}$-based fusion. CP-based fusion also takes some time to process the $3 \times 3$ patches individually, but has shown to be faster than ICA-based fusion in our experiments.

\section{Conclusion}

In this paper, a novel method for image fusion using Chebyshev polynomials as the basis components was introduced. The theory of data fusion, algorithms and motivation of using $\mathrm{CP}$ over other methods was discussed. Performance tests reveal that while processing at a faster rate, $\mathrm{CP}$-based fusion also displays encouraging results in noise corrupted signals and multi-modal images, due to its inherent smoothing property. The use of 2D Chebyshev polynomials for data fusion may also influence other image processing applications.

\section{References}

[1] T. Stathaki, "Image Fusion: Algorithms and Applications",Academic Press, pages 500, 2008.

[2] A. Hyvärinen and P. O. Hoyer, "Emergence of phase and shift invariant features by decomposition of natural images into independent feature subspaces", Neural Computation, Vol. 12, No. 7, pp. 1705-1720, 2000.

[3] N. Mitianoudis and T. Stathaki, "Pixel-based and Region-based Image Fusion schemes using ICA bases", Information Fusion, Vol. 8, No. 2, pp. 131-142, 2007.

[4] N. Amthul, "Image fusion using two dimensional Chebyshev polynomials", MSc Dissertation, Imperial College London, September 2009.

[5] A.T. Benjamin, L. Ericksen, P. Jayawant and M. Shattuck, "Combinational Trigonometry with Chebyshev Polynomials", preprint submitted to Journal of Statistical Planning and Inference, 2008.

[6] J.C. Mason and D.C. Handscomb, "Chebyshev Polynomials", Chapman \& Hall / CRC, Florida, p. 105-141, 2003.

[7] K.B. Dunn and R. Lidl, "Generalizations of the classical Chebyshev polynomials to polynomials in two variables", Czechoslovak Mathematical Journal, Vol. 32, No. 4, p. 516-528, Institute of Mathematics, 1982.

[8] T.H. Koornwinder, "Orthogonal polynomials in two variables which are eigenfunctions of two algebraically independent partial differential operators". I, Nederl. Akad. Wetensch. Proc. Ser. A $77=$ Indag. Math. 36, pp. 48-58, 1974.

[9] L.J. Wojakowski, "Moments of measure orthogonalizing the 2-dimensional Chebyshev polynomials", Banach Center Publications 73, pp. 429-433, 2006.
[10] R. Mukundan, S.H. Ong and P.A. Lee, "Image analysis by Tchebichef moments", IEEE Transactions on Image Processing, Vol. 10 No. 9, pp. 1357-1364, September 2001.

[11] J. Esteban, A. Starr, R. Willetts, P. Hannah and P. Bryanston-Cross, "A review of data fusion models and architecture: towards engineering guidelines, Neural Computing \& Applications, 14: 273-281, SpringerVerlag London Ltd, 2005.

[12] R. Mukundan, "Some computational aspects of discrete orthonormal moments", IEEE Transactions on Image Processing, Vol. 13 No. 8, pp. 1055-1059, August 2004.

[13] M. Cheong and K.S. Loke, "Textile recognition using Tchebichef moments of co-occurrence matrices", Advanced Intelligent Computing Theories and Applications. With Aspects of Theoretical and Methodological Issues, Springer Berlin / Heidelberg, Volume 5226/2008, pp. 1017-1024, September 2008.

[14] M. Alnasser and H. Foroosh, "Rendering synthetic objects in natural scenes", IEEE ICIP 2006, pp. 493-496, October 2006.

[15] D.P. Mandic, D. Obradovic, A. Kuh, T. Adali, U. Trutschel, M. Golz, P. De Wilde, J. Barria, A. Constantinides and J. Chambers, "Data fusion for modern engineering applications: An overview", ICANN 2005, LNCS 3697, pp. 715-721, 2005. 